\title{
Analysis of surgically treated gastric cancers: a tertiary hospital experience in Turkey
}

\author{
Orhan Alimoglu, ${ }^{1}$ Abdullah Sisik, ${ }^{2}$ Tunc Eren, ${ }^{1}$ Suat Aktas, ${ }^{2}$ Fatih Basak, ${ }^{2}$ \\ Metin Leblebici, ${ }^{1}$ Gurhan Bas ${ }^{2}$ \\ ${ }^{1}$ Department of General Surgery, Istanbul Medeniyet University Faculty of Medicine, Istanbul, Turkey \\ ${ }^{2}$ Department of General Surgery, Umraniye Training and Research Hospital, Istanbul, Turkey
}

\begin{abstract}
OBJECTIVE: Gastric cancer (GC) is the fifth most common cancer worldwide, and in Turkey, GC is placed among the 10 most frequently seen cancers. However, analyzing the epidemiology of cancers and improving screening programs for cancers are not still the top priorities for healthcare professionals. This study aims to show distribution of GC based on stages in a tertiary hospital of Istanbul, Turkey.
\end{abstract}

METHODS: All surgically treated GC cases in the General Surgery Department, between January 2009 and January 2014 were retrospectively reviewed. Data including the operation year, the demographic, clinical and histopathological parameters were recorded and statistically analyzed.

RESULTS: The study group consisted of 111 patients. Seventy-four (66.66\%) patients were men, and 37 (33.33\%) patients were women with a mean age of $63.49 \pm 11.83$ years. Stage 3 and 4 were the most frequently observed stages with 62 and 18 cases, respectively. Stage 3 was the most common stage found during the study period $(p<0.05)$. During 5 years of the study period, $G C$ was less frequently located on the cardia $(n=31)$ when compared with other locations $(n=75)$ for GC $(p<0.05)$.

CONCLUSION: Turkey's population has generally advanced stage GC disease. New strategies are needed for achievement of early diagnosis and better outcomes.

Keywords: Gastric cancer; surgery; Turkey.

G astric cancer (GC) is the fifth most common cancer worldwide. Nearly 0.95 million new cases of gastric cancer were detected in the year 2012 worldwide [1].

Gastric cancer was still the fourth most common cancer in the world until 2008, but according to Globocan 2012, it has been the fifth one after lung, breast, colorectal and prostate cancers accounting for $6.8 \%$ of all cancers globally $[1,2]$. Gastric carcinomas have two subgroups according to tumor sites which differ in epidemiologic and pathologic profile, clinical presentation and prognosis such as proximal (cardia) and distal (noncardia) carcinomas $[3,4]$. Most of the gastric adenocarcinomas oc-

Received: June 13, 2015 Accepted: June 20, 2015 Online: September 24, 2015

Correspondence: Dr. Orhan ALIMOGLU. Istanbul Medeniyet Universitesi, Goztepe Egitim ve Arastirma Hastanesi, Genel Cerrahi Klinigi, Istanbul, Turkey.

Tel: +90216 - 5709187 e-mail: orhanalimoglu@gmail.com

(c) Copyright 2015 by Istanbul Northern Anatolian Association of Public Hospitals - Available online at www.kuzeyklinikleri.com 
cur in developing countries. Japan and Korea have the highest rates of gastric carcinoma in the world. $H$. pylori infection, consumption of salty foods and $\mathrm{N}$-nitroso compounds, low intake of fresh fruits and vegetables, smoking, obesity are some common risk factors for gastric cancers.

There was a regular reduction in the global noncardia gastric cancer incidence with different rates among countries. The declining rate was more than $80 \%$ in the USA, while it decreases much less frequently in countries such as Japan, Korea and China [5]. This global reduction may be associated with increased refrigeration, decreased reliance on salted and preserved foods, reduction in chronic $H$. pylori infection, increased application of screening tests in high-risk countries and reduction in smoking. Despite these factors, gastric cardia cancer incidence rates in the USA and Europe have increased. Gastroesophageal reflux disease due to increase in obesity and better distinction from esophageal adenocarcinoma in the light of standardized international classification of the site of cancer are contributing factors for the increase in gastric cardia cancer [6].

Higher incidence of GC is shown in the elderly and with male gender similar to colorectal cancers. GC patients were mostly diagnosed at advanced stages because the signs of disease are often reported too late [7]. Surgical treatment of GC in early stages offer the chance of cure, but in advanced stages, surgery often provides only palliation. Thus, disease stage is a crucial factor in the achievement of successful treatment.

In Turkey, GC is placed among the 10 most common cancers with an increasing incidence [8]. However, analyzing the epidemiology of cancers and improving screening programs for cancers are not still the top priorieties among healthcare professionals.

This study aims to show the distribution of stages of GC in a tertiary hospital of Istanbul, Turkey.

\section{MATERIALS AND METHODS}

All cases of GC who were treated surgically in the General Surgery Department between January 2009 and January 2014 were retrospectively reviewed. Approval for this study was obtained from the Ethics Committee of our institution, and signed informed consent forms were obtained from the patients.

Data were analyzed as for age, gender, tumor TNM stage and tumor location. The cases who were assessed as adenocarcinoma in histopathological evaluations were staged according to the 2012 TNM classification. Stages were grouped from 1 to 4. According to TNM stages "early stage" and "advanced stage" groups were created. The early stage group consisted of stages 1 and 2, whereas the advanced stage group consisted of stages 3 and 4 . The cases were grouped as "others" which were reported

TABLE 1. Characteristics GC patients

atients

Number

Age (mean $\pm S D$ )(years)

Gender

Male/Female (ratio)

Tumor TNM stage

Stage 1, n (\%)

Stage 2, n (\%)

Stage 3, n (\%)

Stage 4, n (\%)

Others
111

$63.49 \pm 11.83$

74/37

62 (55.85)

$18(16.21)$

5 (4.5) 
as lymphoma and gastrointestinal stromal tumor histopathologically. Tumor locations were grouped as "cardia group", "non-cardia group" and "remnant stomach" group.

All cases were separated and analyzed according to years in which cancer was diagnosed. The differences between cases of each year by age, gender, TNM stage, early or advanced stage groups and location were evaluated. We also analyzed these data according to their proportional distributions and annual changes.

The variables were statistically analyzed in consideration of their numbers and proportional values since the number of some variables were very few. Statistical calculations were performed with use of the IBM SPSS 22 (IBM SPSS, USA). Normallydistributed continuous variables were expressed as mean \pm standard deviation (SD). Cathegorical variables were expressed as frequencies and percentages. Chi-square, Fisher's exact test and Yates Continuity Correction test were used for the comparisons of continuous parametric variables. Student's test was used for the comparisons of parametric variables with normal distribution. The statistical results were presented with a $95 \%$ confidence interval. The differences were considered statistically significant when $p$ value was $<0.05$.

\section{RESULTS}

Hundred and eleven patients with gastric cancer were operated during the study period. Seventyfour $(66.66 \%)$ patients were male and 37 (33.33\%) were female. The mean age of the study population was $63.49 \pm 11.83$ years. Annual distribution of the patients according to their gender and ages are shown in Figures 1 and 2.

Annual number of patients during the study period varied $(n=12,22,30,24$ and 23 for the years 2009, 2010, 2011, 2012, and 2013, respectively) Disease stage analyses of patients who were assessed as adenocarcinoma in histopathological examinations revealed that stages 3 and 4 were the most determined stages with 62 , and 18 cases, respectively (Table 1). Examination of the annual distribution of stages did not show significant differences while

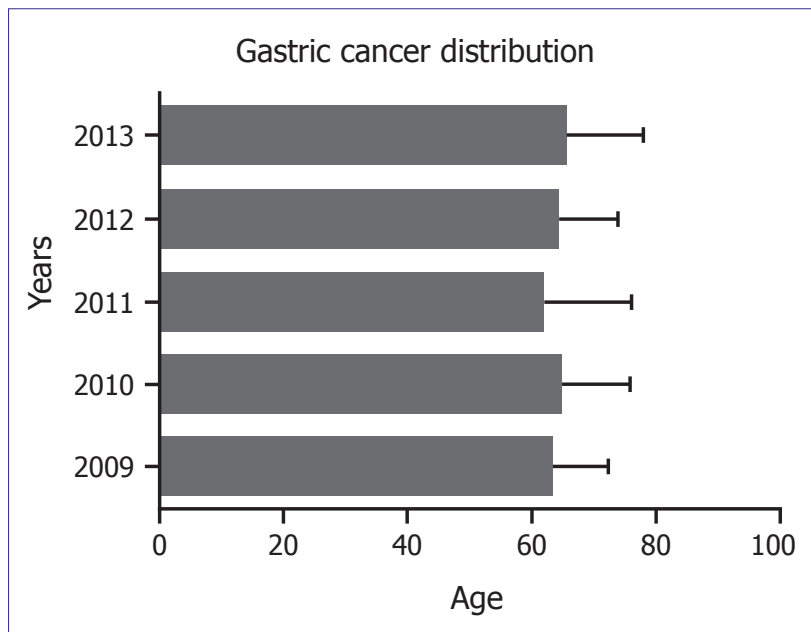

FIGURE 1. Annual age distribution for GC.

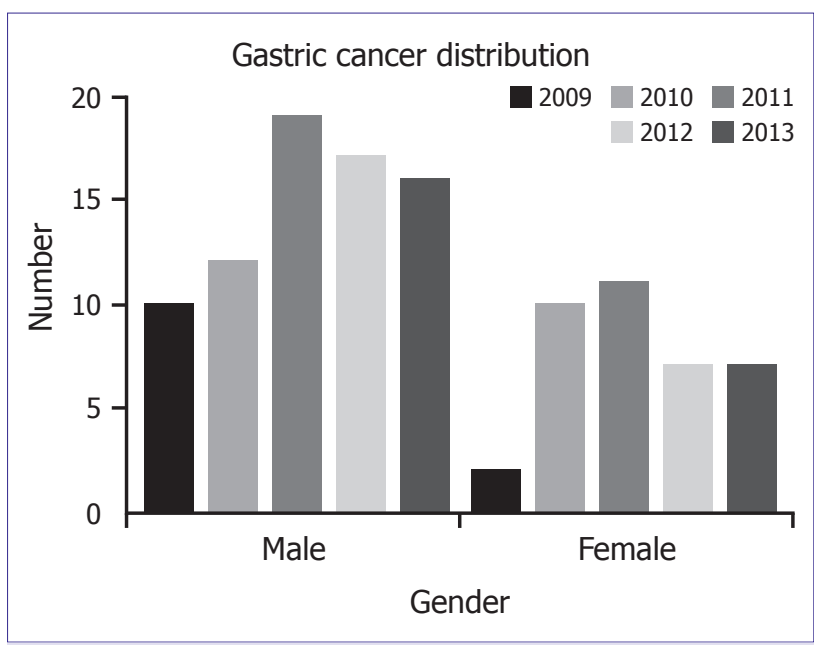

FIGURE2. Annual gender distribution for GC.

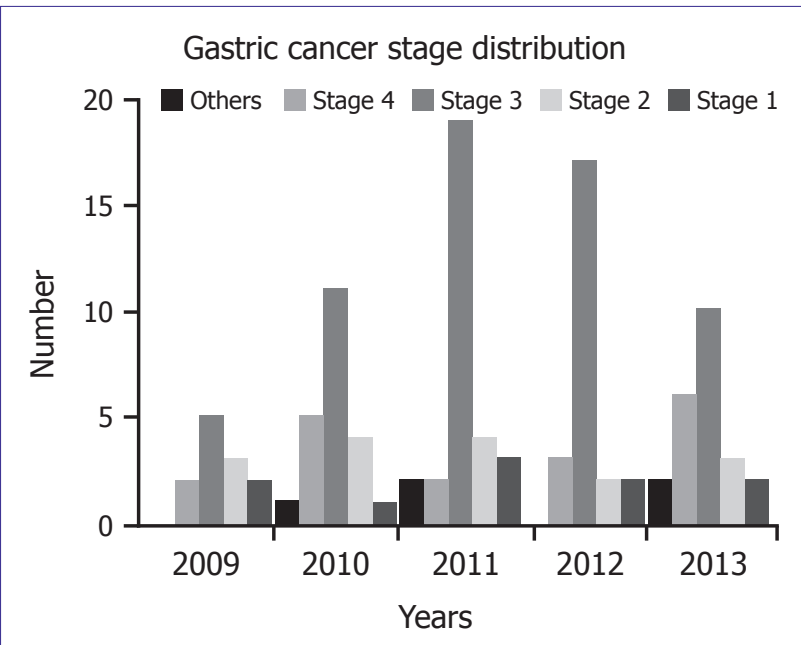

FIGURE 3. Annual stage distribution for GC. 
analyzing proportional values yielded statistically significant results $(\mathrm{p}<0.001)$ (Table 2, Figure 3).

Stage 3 was the most commonly seen stage during study period. Early and also advanced stages were also examined annually, and no significant difference was found $(p=0.6057)$. Still proportional values were statistically significant $(\mathrm{p}=0.0012)(\mathrm{Ta}$ ble 3). Advanced stages were mostly detected annually during study period.
GC was detected most frequently on locations other than cardia during 5 years of the study period (cardia, $n=31$, and non-cardia, $n=75)(p=0.3073$, $\mathrm{p}<0.001)$ (Table 4).

\section{DISCUSSION}

Cancer is one of the major public health problems in Turkey. It has been estimated that within the next 20-30 years cancer will take the highest death

TABLE2. Annual TNM stage distribution for GC

\begin{tabular}{|c|c|c|c|c|c|c|c|c|c|c|c|}
\hline & \multicolumn{2}{|c|}{2009} & \multicolumn{2}{|c|}{2010} & \multicolumn{2}{|c|}{2011} & \multicolumn{2}{|c|}{2012} & \multicolumn{2}{|c|}{2013} & \multirow[t]{2}{*}{$\mathrm{p}$} \\
\hline & $\mathrm{n}$ & $\%$ & $n$ & $\%$ & $\mathrm{n}$ & $\%$ & $n$ & $\%$ & $\mathrm{n}$ & $\%$ & \\
\hline Stage 1 & 2 & 16.67 & 1 & 4.76 & 3 & 10.71 & 2 & 8.33 & 2 & 9.52 & \\
\hline Stage 2 & 3 & 25 & 4 & 19.05 & 4 & 14.29 & 2 & 8.33 & 3 & 14.29 & \\
\hline Stage 3 & 5 & 41.67 & 11 & 52.38 & 19 & 67.86 & 17 & 70.83 & 10 & 47.62 & $<0.0001$ \\
\hline Stage 4 & 2 & 16.67 & 5 & 23.81 & 2 & 7.14 & 3 & 12.5 & 6 & 28.57 & \\
\hline Total & 12 & & 21 & & 28 & & 24 & & 21 & & \\
\hline
\end{tabular}

TABLE 3. Annual early and advanced stage distribution of GC

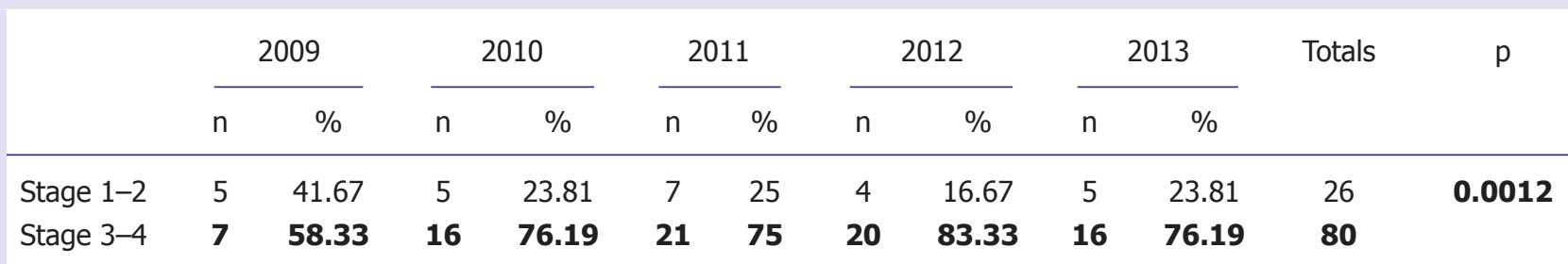

TABLE 4. Annual tumor location distribution for GC

\begin{tabular}{|c|c|c|c|c|c|c|c|c|c|c|c|c|}
\hline & \multicolumn{2}{|c|}{2009} & \multicolumn{2}{|c|}{2010} & \multicolumn{2}{|c|}{2011} & \multicolumn{2}{|c|}{2012} & \multicolumn{2}{|c|}{2013} & \multirow[t]{2}{*}{ Total } & \multirow[t]{2}{*}{$\mathrm{p}$} \\
\hline & $\mathrm{n}$ & $\%$ & $\mathrm{n}$ & $\%$ & $\mathrm{n}$ & $\%$ & $\mathrm{n}$ & $\%$ & $\mathrm{n}$ & $\%$ & & \\
\hline Cardia & 2 & 16.67 & 4 & 18.18 & 9 & 30 & 9 & 37.5 & 7 & 30.44 & 31 & \\
\hline Non-cardia & 10 & 83.33 & 18 & 81.82 & 20 & 66.67 & 12 & 50 & 15 & 65.22 & 75 & $<0.0001$ \\
\hline Remnant & 0 & 0 & 0 & 0 & 1 & 3.33 & 3 & 12.5 & 1 & 4.34 & 5 & \\
\hline Total & 12 & & 22 & & 30 & & 24 & & 23 & & 111 & \\
\hline
\end{tabular}


toll $[9,10]$. Epidemiological evaluations of GC, and all other cancer types are important in determining the treatment, early diagnosis and the development of screening programs. In Far East, screening programs were developed for GC, which is common in that region, and early diagnosis and improved survival from GC are achieved. In most of the studies performed, GC patients were most frequently in advanced age and of male gender.

As the geriatric population increases in the world, GC continues to be an important public health issue currently, and will be so in the future. Despite this, the impact of some regional differences about age of the patients have been evaluated as for the presence of age-dependent demographic characteristics. In our study, most of the patients were older ages with a mean age of $63.49 \pm 11.83$ years. Male gender dominancy was determined, and this data is consistent with the literature [11].

Disease stage is one of the most important prognostic factors for GC patients. Patients diagnosed in early stages have more improved outcomes than those in their advanced stages. Different screening programmes using endoscopic procedures, tumor marker analysis, etc. have been applied in different countries for the diagnosis of cancer, and prediction of its stage $[12,13]$. In the literature, diverse distributions of disease stages have been reported from various regions based on environmental and regional differences. As mentioned above early disease stages of GC has been determined in the Far East. Despite limited information in the literature, data on distribution of disease stage of GC in Turkey shows that most of the patients have advanced stage diseases [14]. We found that stage 3 is the most common stage and analysis of annual change didn't show any difference in its incidence. Almost $80 \%$ of GC patients have advanced stages.

The dietary habits in Turkey have broadly changed during recent years. Intake of fruits and vegetables decreased, while that of animal protein, meat, sugar refined cereals increased. Becoming an industrial society with a predisposition to a western style nutrition and modification of the lifestyle may be the underlying cause of of this alteration.
In Turkey, there are no examples of communitybased screening programmes covering specific target groups, except for some sporadic pilot studies. Parallel to joint deficiencies in screening protocol and detection of cancers at an early stage, it is widely accepted that cancer cases are diagnosed at an advanced stage in Turkey, causing early and preventable deaths. Current study suggests that healthcare management professionals must develop new strategies about screening programmes especially in GC.

Recently, the prevalence of adenocarcinoma of the esophagus and cardia increased along with a decrease in distal gastric cancers in developed countries. The data from developing countries about this alteration is very scarce. Some studies showed different results concerning anatomical location of gastric adenocarcinoma for the west and east parts of Turkey. In our study, non-cardia location was predominantly determined and its predominancy didn't change annually $[7,8]$.

This study has several potential limitations. First, the number of patients is not enough to make definitive recommendations. Multicenter analysis may be more valuable. Another limitation is retrospective design of the study. Randomized- controlled prospective studies would be more beneficial about clarification of this issue.

In conclusion, Turkey's population has advanced stage GC generally. New strategies are needed for earlier detection of GC, and achievement of better outcomes.

Conflict of Interest: No conflict of interest was declared by the authors.

Financial Disclosure: The authors declared that this study has received no financial support.

\section{REFERENCES}

1. Ferlay J, Soerjomataram I, Ervik M, et al. GLOBOCAN 2012 v1.0, Cancer Incidence and Mortality Worldwide: IARC CancerBase No. 11 [Internet]. Lyon, France: International Agency for Research on Cancer 2013.

2. Parkin DM, Pisani P, Ferlay J. Global cancer statistics. CA Cancer J Clin 1999;49:33-64.

3. Jackson C, Mochlinski K, Cunningham D. Therapeutic options in gastric cancer: neoadjuvant chemotherapy vs postoperative 
chemoradiotherapy. Oncology (Williston Park) 2007;21:10847; discussion 1090, 1096-8, 1101.

4. Cunningham D, Allum WH, Stenning SP, Thompson JN, Van de Velde CJ, Nicolson M, et al. Perioperative chemotherapy versus surgery alone for resectable gastroesophageal cancer. N Engl J Med 2006;355:11-20.

5. Jemal A, Siegel R, Ward E, Hao Y, Xu J, Thun MJ. Cancer statistics, 2009. CA Cancer J Clin 2009;59:225-49.

6. Fock KM. Review article: the epidemiology and prevention of gastric cancer. Aliment Pharmacol Ther 2014;40:250-60.

7. Mohandas KM, Jagannath P. Epidemiology of digestive tract cancers in India. VI. Projected burden in the new millennium and the need for primary prevention. Indian J Gastroenterol 2000;19:74-8.

8. Gultekin M, Boztas G ed. Turkish Ministry of Health Public Health Agency Cancer Statistics 2014.

9. Ministry of Health: National Burden of Disease and Cost Effectiveness Study. Turkish Ministry of Health, Ankara 2004.
10. Tuncer $\mathrm{M}$. The national cancer control program: short, medium and long term action plan from the global perspective. In: Tuncer M. (ed.) Cancer Control in Turkey. Ministry of Health Publication, Ankara 2008:740.

11. Bor S, Vardar R, Ormeci N, Memik F, Suleymanlar I, Oguz D, et al. Prevalence patterns of gastric cancers in Turkey: model of a developing country with high occurrence of Helicobacter pylori. J Gastroenterol Hepatol 2007;22:2242-5.

12. Sisik A, Kaya M, Bas G, Basak F, Alimoglu O. CEA and CA 19-9 are still valuable markers for the prognosis of colorectal and gastric cancer patients. Asian Pac J Cancer Prev 2013;14:4289-94.

13. Sankaranarayanan R. Screening for cancer in low- and middleincome countries. Ann Glob Health 2014;80:412-7.

14. Erturk MS, Ciçek Y, Ersan Y, Saribeyoğlu K, Doğusoy G, Erginoz E. Analysis of clinicopathological prognostic parameters in adenocarcinoma of the gastric cardia. Acta Chir Belg 2003;103:611-5. 